\title{
Mathematical modeling of ignition of coal-water slurries particles coated with water film
}

\author{
V. G. Afanaseva ${ }^{1, *}$ \\ ${ }^{1}$ National Research Tomsk Polytechnic University, 634050 Tomsk, Russia
}

\begin{abstract}
The results of the mathematical modeling of heat and mass transfer processes occurring jointly with ignition of coal-water slurries particles covered with a water film are presented. It is shown that water film thickness can have a significant effect on the ignition dynamics. In addition, it was found out that the main fuel layer is heated to the evaporation temperature during water film evaporation. As a result, following processes of thermal preparation (evaporation of moisture, thermal decomposition) occur faster.
\end{abstract}

\section{Introduction}

Presently, climate change problem is particularly acute. The main cause of the current global warming trend is increasing concentration of carbon dioxide in the atmosphere, resulting in the greenhouse effect. In Russia, about $70 \%$ of the carbon dioxide evolution is caused by the fossil fuel combustion in thermal power plants. The highest level of emissions is observed during coal combustion, therefore, the first thing we have to do is develop clean coal technologies to minimize the negative impact on the environment.

One of such technological solutions that ensure safety and efficiency of coal use is the technology of combustion it in the form of coal water slurry (CWS) [1-4].

The large-scale inmplementation of CWS in the energy sector is hampered by many factors, one of which is insufficient study of the processes occurring during the CWS thermal preparation for ignition. Experimental studies of CWS combustion in boiler units require theoretical studies of the ignition and burning regularities, which are necessary for solving the organization of suspension combustion problems.

At the time of CWS spraying in furnace volume of a boiler, two classes of CWS drops form: the first class includes partially dried CWS particles, the second class contains more watered drops with a near-surface water film [5]. The ignition characteristics of the latter class are currently insufficiently studied, but it is clear that the presence of a water film can have an impact on the ignition of CWS particles due to the high endothermic evaporation and heat capacity of water [6].

Thus, the purpose of the article is mathematical modeling of the heat and mass transfer processes occurring jointly with ignition of coal-water slurries particles covered with a water film.

\footnotetext{
* Corresponding author: ssyrodoy@yandex.ru
} 


\section{Formulation of the problem}

The mathematical formulation of the problem, the corresponding stated higher of physical model is a non-stationary system of differential equations in partial derivatives:

CWS particle has a polyhedral shape that depends on many factors and can vary randomly, therefore, the particle was observed in the spherical form for simplification [7].

At the initial time moment $(t=0)$ the CWS particle enters the gas environment, the temperature of which far exceeds the particle temperature. The particle absorbs the gas environment heat through convection and radiation, as a result the evaporation of the water film begins. It is taken that thermal radiation is absorbed only by the water film [6].

After the water film evaporation, further warming of the main fuel layer begins, the warming is followed by pore moisture evaporation and thermal decomposition of the fuel combustible matter with volatile content. The processes run before the volatiles igniting near the particle. It is taken that combustion of volatiles includes the following reactions:

$$
\begin{gathered}
2 \mathrm{H}_{2}+\mathrm{O}_{2} \rightarrow 2 \mathrm{H}_{2} \mathrm{O}+115.5 \mathrm{~kJ} / \text { mole' } \\
\mathrm{CH}_{4}+2 \mathrm{O}_{2} \rightarrow \mathrm{CO}_{2}+2 \mathrm{H}_{2} \mathrm{O}+892 \mathrm{~kJ} / \text { mole' } \\
2 \mathrm{CO}+\mathrm{O}_{2} \rightarrow 2 \mathrm{CO}_{2}+285 \mathrm{~kJ} / \text { mole }
\end{gathered}
$$

The mathematical formulation of the problem includes non-stationary differential equations in partial derivatives, which are shown below.

The energy equation for the CWS particle:

$$
c_{p}(T) \rho_{p}(T) \frac{\partial T}{\partial t}=\frac{1}{r^{2}} \frac{\partial}{\partial r}\left(r^{2} \lambda_{p}(T) \frac{\partial T}{\partial r}\right)+\frac{1}{r^{2} \sin \varphi} \frac{\partial}{\partial \varphi}\left(\sin \varphi \lambda_{p}(T) \frac{\partial T}{\partial \varphi}\right)-q_{t h} W_{t h}-Q_{e v} W_{e v}
$$

The thermo-physical properties of CWS particle $\left(c_{p}, \rho_{p}, \lambda_{p}\right)$ were evaluated by position of evaporation front and were calculated taking into account volume ratio of CWS particle components.

There is the boundary condition of the fourth kind at the interface of the "CWS particlewater film", taking into account the radiation heat transfer:

$$
r=r_{p}, 0<\varphi<\pi:\left\{\begin{array}{c}
\lambda_{p}(T) \frac{\partial T_{p}}{\partial r}=\lambda_{\Sigma} \frac{\partial T_{w}}{\partial r} \\
T_{p}=T_{w}
\end{array}\right.
$$

The energy equation for the water film $\left(t>0, r_{p}<r<r_{w}, 0<\varphi<\pi\right)$ :

$$
c_{w} \rho_{w} \frac{\partial T}{\partial t}=\frac{1}{r^{2}} \frac{\partial}{\partial r}\left(r^{2} \lambda_{\Sigma} \frac{\partial T}{\partial r}\right)+\frac{1}{r^{2} \sin \varphi} \frac{\partial}{\partial \varphi}\left(\sin \varphi \lambda_{\Sigma} \frac{\partial T}{\partial \varphi}\right)
$$

The radiation absorption by water film was adopted in accordance with the Rosseland radiation model [8]:

$$
\lambda_{\Sigma}=\lambda_{w}+\frac{16 \sigma T^{3}}{3 a_{R}}
$$

There is the boundary condition of the fourth kind at the interface of the "water film-gas environment", taking into account the radiation heat transfer and the thermal effect of vaporization: 


$$
r=r_{w}, 0<\varphi<\pi:\left\{\begin{array}{c}
\lambda_{\Sigma} \frac{\partial T_{w}}{\partial r}=\lambda_{g} \frac{\partial T_{g}}{\partial r}-Q_{e v} W_{e v} \\
T_{w}=T_{g}
\end{array}\right.
$$

In the equations (1) and (4) the mass evaporation rate was calculated from the expression:

$$
W_{e v}=W_{0} \cdot \exp \left[\frac{Q_{e v} \mu\left(T-T_{f}\right)}{R T T_{f}}\right]
$$

Thermal decomposition of the fuel combustible matter was described by chemical kinetics equation:

$$
\left\{\begin{array}{c}
\frac{\partial \eta}{\partial t}=(1-\eta) k_{t h} \exp \left(\frac{-E_{t h}}{R T}\right) \\
W_{t h}=(1-\eta) \cdot k_{t h} \cdot \rho(T) \cdot \exp \left(\frac{-E_{t h}}{R T}\right)
\end{array}\right.
$$

The energy equation for the gas environment:

$$
c_{g} \rho_{g} \frac{\partial T}{\partial t}=\frac{1}{r^{2}} \frac{\partial}{\partial r}\left(r^{2} \lambda_{g} \frac{\partial T}{\partial r}\right)+\frac{1}{r^{2} \sin \varphi} \frac{\partial}{\partial \varphi}\left(\sin \varphi \lambda_{g} \frac{\partial T}{\partial \varphi}\right)+Q_{i} W_{i}
$$

In the equation (5) the rate of chemical reactions was calculated from the mathematical expression of the Arrhenius law:

$$
W_{i}=k_{i} C_{i}\left(1-\sum_{i=1}^{4} C_{i}\right) \rho_{g} \cdot \exp \left(\frac{-E_{i}}{R T}\right)
$$

The boundary condition at the gas frontier line:

$$
r=r_{g}, 0<\varphi<\pi: \frac{\partial^{2} T}{\partial r^{2}}=0
$$

The volatile substances concentration dependence of coordinates and time is described by the diffusion equation $\left(t>0, r_{p}<r<r_{g}, 0<\varphi<\pi\right)$ :

$$
\frac{\partial C_{i}}{\partial t}=\frac{D_{i}}{r^{2}}\left[\frac{\partial}{\partial r}\left(r^{2} \frac{\partial C_{i}}{\partial r}\right)+\frac{1}{\sin \varphi} \frac{\partial}{\partial \varphi}\left(\sin \varphi \lambda_{g} \frac{\partial C_{i}}{\partial \varphi}\right)\right]
$$

The equations (1-6) were solved with the following boundary conditions:

$$
\begin{gathered}
\varphi=0,0<r<r_{g}: \frac{\partial T}{\partial \varphi}=0 \\
\varphi=\pi, 0<r<r_{g}: \frac{\partial T}{\partial \varphi}=0 \\
r=r_{p}, 0<\varphi<\pi:\left\{\begin{array}{c}
\frac{\partial C_{i}}{\partial r}=-\frac{W_{i}}{\rho_{g} D_{i}} \\
W_{i}=V_{i}(1-\eta) \rho(T) \cdot \exp \left(\frac{-E_{t h}}{R T}\right) \\
r=r_{g}, 0<\varphi<\pi: \frac{\partial^{2} C_{i}}{\partial r^{2}}=0 \\
\varphi=0, r_{p}<r<r_{g}: \frac{\partial C_{i}}{\partial \varphi}=0
\end{array}\right.
\end{gathered}
$$




$$
\varphi=\pi, r_{p}<r<r_{g}: \frac{\partial C_{i}}{\partial \varphi}=0
$$

The designations: $T 0$ - initial temperature of the particle, $\mathrm{K} ; r p$ - radius of the CWS particle, $\mathrm{m} ; r w$ - radius of the CWS particle with the water film, $\mathrm{m} ; r g$ - radius from center of the CWS particle to boundary of the gas environment, $\mathrm{m} ; \lambda p$ - coefficient of thermal conductivity of the CWS particle, $\mathrm{W} /(\mathrm{m} \cdot \mathrm{K}) ; \mathrm{cp}$ - heat capacity of the CWS particle, $\mathrm{J} /(\mathrm{kg} \cdot \mathrm{K})$; $\rho p$ - density of the CWS particle, $\mathrm{kg} / \mathrm{m}^{3} ; \lambda w$ - coefficient of thermal conductivity of water, $\mathrm{W} /(\mathrm{m} \cdot \mathrm{K}) ; c w$ - heat capacity of water, $\mathrm{J} /(\mathrm{kg} \cdot \mathrm{K}) ; \rho w-$ density of water, $\mathrm{kg} / \mathrm{m}^{3} ; \lambda g-$ coefficient of thermal conductivity of the gas, $\mathrm{W} /(\mathrm{m} \cdot \mathrm{K}) ; c g$ - heat capacity of the gas, $\mathrm{J} /(\mathrm{kg} \cdot \mathrm{K}) ; \rho g$ - density of the gas, $\mathrm{kg} / \mathrm{m}^{3} ; W e v$ - mass rate of water evaporation, $\mathrm{kg} /\left(\mathrm{m}^{2} \cdot \mathrm{s}\right)$; $Q e v$ - thermal effect of the water evaporation, $\mathrm{J} / \mathrm{kg} ; \mathrm{Tf}$ - temperature of ice point, $\mathrm{K} ; \mathrm{W} 0$ mass rate of water evaporation for temperature $T f, \mathrm{~kg}^{2}\left(\mathrm{~m}^{2} \cdot \mathrm{s}\right) ; \mu$ - molar mass of water, $\mathrm{kg} /$ mole; $\mathrm{R}$ - universal gas constant, $\mathrm{J} /($ mole $\cdot \mathrm{K}) ; Q i$ - thermal effect of a chemical reaction, $\mathrm{J} / \mathrm{kg} ; k i$ - preexponent of chemical reaction $1 / \mathrm{s} ; E i$ - activation energy of a chemical reaction, $\mathrm{J} / \mathrm{mole} ; D i$ - binary diffusion coefficient, $\mathrm{m}^{2} / \mathrm{s} ; \sigma-$ Stefan-Boltzmann constant, $\mathrm{W} \cdot \mathrm{m}^{-2} \cdot \mathrm{K}^{-4}$; $a R$ - Rosseland mean absorption coefficient, $1 / \mathrm{m}, V i$ - volatile yield.

\section{The results}

At the time of CWS spraying in furnace volume of a boiler, a lot of particles are formed, the sizes of which differ from each other. The thickness of the water film which is coated the CWS particles can also assume a different value. Accordingly, it can be said that the water film thickness is a significant factor affecting on the characteristics of the CWS particles ignition. At the present time the degree of such influence is poorly known.

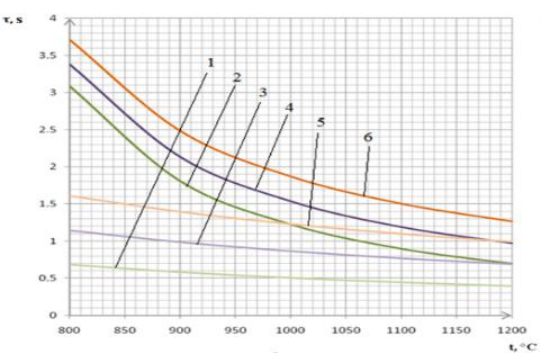

a

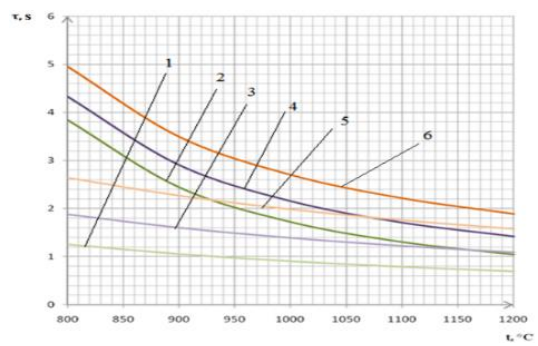

b

Fig. 1. The dependence of the water film evaporation times and the ignition delay times of the ambient temperature with the water film thickness of $0.12 \cdot 10^{-3} \mathrm{~m}$ (a) and $0.12 \cdot 10^{-3} \mathrm{~m}$ (b) at a rate (d) particles: $1,2-\mathrm{d}=2 \cdot 10^{-3} \mathrm{~m} ; 3,4-\mathrm{d}=3 \cdot 10^{-3} \mathrm{~m} ; 5,6-\mathrm{d}=4 \cdot 10^{-3} \mathrm{~m}$.

Fig. 1 illustrates the time dependence of the CWS particle ignition delay and the water film evaporation, with variation of particle diameter and film thickness. Analysis of dependences revealed that the furnace volume temperature increase reduces the ignition delay time. It can be noted that the diameter increase leads to the induction period decrease. So the diameter increase from 2 to $4 \mathrm{~mm}$ results in the ignition delay time increase by $30 \%$ (from 3 to $5 \mathrm{~s}$ ), at $\mathrm{T}_{\mathrm{e}}=1073 \mathrm{~K}$.

The film thickness increase (Fig. 1, b) leads to an elongation of the induction period due to the high endothermic evaporation and heat capacity of water.

Fig. 2 shows the time dependence of the evaporation time of the water film and the ignition delay time of CWS particles coated with a water film, which has different thicknesses. It can be noted that increasing the film thickness from 0.12 to $0.4 \mathrm{~mm}$ leads to the ignition process slowing down by $50 \%$. 
Analysis of the dependences in Fig. 2 shows that the evaporation of a water film has a significant effect on the thermal preparation for the CWS particles ignition.

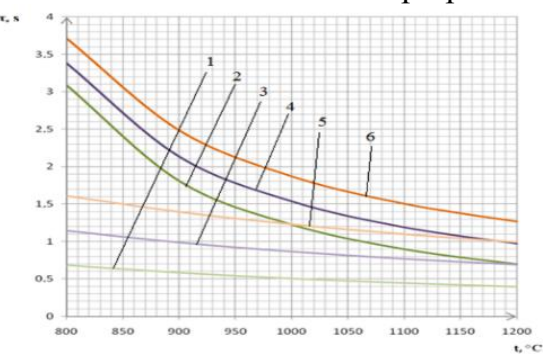

a

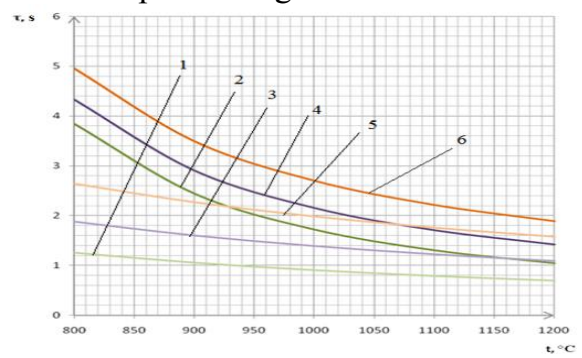

b

Fig. 2. The dependence of the water film evaporation times and the ignition delay times of the ambient temperature with the particle diameter of $2 \cdot 10^{-3} \mathrm{~m}$ (a) and $3 \cdot 10^{-3} \mathrm{~m}$ (b) at a film thickness $(\Delta): 1,2-\Delta=0.12 \cdot 10^{-3} \mathrm{~m} ; 3,4-\Delta=0.25 \cdot 10^{-3} \mathrm{~m} ; 5,6-\Delta=0.4 \cdot 10^{-3} \mathrm{~m}$.

It can be noted that the film evaporation time for almost the entire temperature range is up to $60 \%$ of the induction period, it is due to the fact that during the evaporation of the water film the main fuel layer is heated to the evaporation temperature. For that reason, the following stages of the thermal preparation of the CWS particle (without a film) and ignition proceed much faster.

\section{Conclusion}

According to the results of the computational experiments it was established that the nearsurface water film can have a significant effect on the characteristics and conditions of the CWS particles ignition. It is shown that the film thickness increase leads to an elongation of the induction period. Increase of the ambient temperature and film thickness leads to increase of the evaporation process role on the integral characteristics of the ignition process. When the water film evaporates, the main fuel layer is heated to the evaporation temperature. So, the thermal preparation processes (evaporation of moisture in the CWS particle, thermal decomposition) proceed more quickly.

The research has been carried out at the expense of the grant of the Russian Scientific Foundation (project No 15-19-10003).

\section{References}

1. O. Erenkov, I. Galanina, E. Urazova Misyura, S. L. Elistratov, R. A. Dekhtyar, Perspective development of science, engineering and technology: Materials of the Third International scientifically-practical conference 1, 397 (2013)

2. O. Kalanikhin, Ecological safety 2, 78 (2013)

3. A. Kuznetsov, I. Tokarev, Modern science 1, 32 (2010)

4. A. Kuznetsov, I. Tokarev, Modern science 1, 21 (2011)

5. J. Senchurova, V. Murko, V. Fedyaev, D. Dziuba, E. Pusirev, The Tomsk Polytechnic University news 312, 38 (2008)

6. S. Syrodoy, N. Gutareva, R. Taburchinov, MATEC Web Conf. 72, 01109 (2016)

7. E. Flek, The Journal Omsk Scientific Bulletin 6, 102 (2017)

8. R. Siegel, Thermal radiation heat transfer (Mir, Moscow, 1975) [in Russian] 